\title{
TECHNICAL VIABILITY STUDY FOR BEHAVIORAL MONITORING OF WILDLIFE ANIMALS IN DOÑANA An 802.15.4 Coverage Study in a Natural Park
}

\author{
M. Dominguez-Morales, A. Jimenez-Fernández, D. Cascado-Caballero, \\ A. Linares-Barranco, R. Paz, G. Jiménez-Moreno \\ Robotic and Technology of Computers Lab, University of Seville, ETSII Av. Reina Mercedes s/n, Seville, Spain \\ alinares@atc.us.es \\ R. Soriguer \\ Biological Station of Doñana, CSIC, Av. Americo Vespucio. EDB-CNM, Seville, Spain
}

Keywords: Wireless sensor networks, WSN, XBee, ZigBee, 802.15.4, Motes, Wireless coverage, Wild animals, Doñana.

\begin{abstract}
The study and monitoring of wildlife and in semi-freedom has always been a subject of great interest. In recent years the technology allows to design low cost systems that facilitate these tasks: microcontrollers, low-power wireless networks, sensors. GPS, satellite and VHF has been used for position tracking and localization of wildlife. Our aim is to design a low-cost system for local monitoring of wildlife (collar or harness) with local memory and remote access. It will use multiples sensors for behavioral and health monitoring and fuse the processed information locally to reduce the stored data, but allowing to be sporadically transmitted through wireless networks. This collar will be based on an embedded low-power microcontroller with 802.15.4 transceiver and a set of sensors to provide data (activity and health) of the animal under monitoring: accelerometers, compass, humidity, temperature, light, microphone, heart rhythm. This collar is called mote in wireless sensors network (WSN) terminology. Only when one mote is closer to an 802.15.4 mote and under request, the collar will dump the information to a host database server through the 802.15.4 network. In this paper we present a viability study of the WSN for Donana Natural Park for different mote transmission powers, frequencies and distances for coverage. ZigBee and XBee mote has been proven.
\end{abstract}

\section{INTRODUCTION}

The monitoring of animal life can be classified into two groups: monitoring wildlife and livestock operations monitoring extensive. During the second half of the twentieth century basically three wildlife tracking methods were used: Haller (2001), Markham (2008), Findholt (1996) and Mech (2002) by VHF tracking, by GPS and by Satellite. The traditional VHF system collapse as soon as it started using multiples collars because of the scarcity of frequencies assigned, which began to codify the signs that each collars forward to optimize localization, as in Allen (2009) work. Satellite localization mechanisms (Argos in particular) are so expensive that only migratory animals were used in the continent, as in Mech (2002) and Vodafone references. Back in the nineties years, localization mechanisms were implemented based on fixed nodes covering a wide area by triangulation (without using GPS) with a precision of 50 meters. This was subsequently improved by GPS obtaining a higher precision, see Rempel (1995). Some of the systems referenced are the ZebraNet that apart of using GPS to implement localization, it includes other sensors to detect the monitored animal activity. However, the main characteristics of our system is that it stores the data collected locally and that these may be transferred between different collars until they are collected by the researcher.

The ZebraNet system, see Juang (2002), describes mechanisms for data collection from different sensors in order to process the information off-line. This allows finding patterns in information that can be associated to the various activities of 
animal: eat, hunt, sleep,... The possibility that this pattern recognition can be performed locally on each collar on the animal is very attractive because it reduces the transmission time and the information post processing.

In this paper we present the results of a viability study of applying WSN for these collars in the Doñana Natural Park. The viability study is focused on 802.15.4 networks with different power transmissions and two different frequencies: $868 \mathrm{MHz}$ and $2.4 \mathrm{GHz}$.

Next section presents a review of 802.15.4 WSN, focusing on ZigBee and XBee standards. Section 3 presents the scenario for the viability study. Then in section 4 we present some results and finally we present the conclusions and future work in section 5 .

\section{WSN TECHNOLOGIES}

802.15.4 (Zigbee Alliance web page: http://www.zigbee.org) is the most representative example of Wireless Sensor Networks (WSN). It is a standard that covers several PHY layers and one MAC layer, aiming to give low rate network service to devices with low capacity batteries. Expected battery operation time is 4 to 6 months for button batteries. Data rates were defined to $250 \mathrm{Kbps}$ at maximum, but lower data rates are possible by choosing the appropriate PHY layer or changing the MAC parameters. Communications security is ensured by AES encryption and coexistence is solved by channel selection and features such as quality of service or noise carrier sensing help to avoid busy channels. Location information is possible through triangulation of RSSI (as implemented in Chipcon CC2431 chips), as in Merrett (2008). Network topologies allowed are centralized (star topology with a network master) or Ad-Hoc (peer to peer communications without master). In star topology, beacon enabled communications make possible to reserve transmission slots, guaranteeing data rates and making soft real time applications possible.

802.15.4 is the base of Zigbee that tries to give a complete solution (with more layers and profiles) to low-rate, low-power personal area networks. A profile is a set of protocols and definitions (such as type of messages, IDs, etc) that must be implemented in case of adopting a specific profile in order to achieve interoperability between devices. One example of this is the recently approved Zigbee Health Care Profile, which offers an open standard for health monitoring and management devices, offering a wide variety of health-care oriented services and protocols.

\section{TESTING SCENARIO}

Doñana National Park, see Doñana (1994) reference, in Andalusia occupies the right bank of the Guadalquivir River at its estuary on the Atlantic Ocean. It is notable for the great diversity of its biotopes, especially lagoons, marshlands, fixed and mobile dunes, scrub woodland and maquis. The faunal inventory includes 8 species of fish, 10 amphibians, 19 reptile, 30 mammal and 360 bird. It is one of the largest heronries in the Mediterranean region and is the wintering site for more than $500 \mathrm{~K}$ water fowl each year. Doñana National Park has been a testing ground for conservation in Spain and has become very well known throughout Europe due to the controversies faced there and the innovative management approaches that have been taken. It is the only protected area that is not only a National Park but also a Ramsar site, a Biosphere Reserve as well as a European Community Special Protection Area. It is also known as the site which triggered the foundation of WWF in 1961.

The Doñana Scientific Reserve (DBR) is made up by two estates with a surface area of 10,000 hectares, included in the 50,000 hectares of Doñana National Park.

This area includes 4 large ecosystems: beaches, dunes, scrubland, and marshland. The fauna includes 41 species of ants, 7 of freshwater fish, 30 of estuary fish, 11 of amphibians, 19 of reptiles and 20 of mammals. DBR has an important infrastructure for scientific research: accommodation rooms, field laboratories, a fleet of 4 wheel-drive cars, horses, boats; and personnel.

In april 2006, the Interministerial Commission of Science and Technology (CICYT) part of the Ministry of Education and Science approved the recognition as Singular Scientific and Technological Infrastructure (ICTS) to the Scientific Reserve of Doñana. ICTS was created with two objectives: (a) providing modern communications and scientific equipments infrastructure to the Reserve in order to allow the standardization and automation of monitoring natural processes, and for developing research activities that could not be possible without the ICTS. And (b) providing access to these facilities and welcoming to the scientific community to develop research activities.

The ICTS is equipped with an extensive audiovisual network for monitoring; a meteorology 
and microclimatology monitoring network; atmospheric measurements equipments; hydrology and limnology; geomorphology; flora and vegetation natural process monitoring; wildlife census; flow of water and $\mathrm{CO} 2$; knowledge of the land on which sits the vegetation; geolocalization through various schemes like FindAve (GPRS and Wifi), RTLS over Wifi, RFID, ultrasound or harmonic radar detectors. Several 802.11 antennas warranty coverage for wireless internet access inside the Reserve.

In order to study the viability of the communications for wildlife monitoring, two different 802.15.4 frequencies has been tested in this work: $2.4 \mathrm{GHz}$ and $868 \mathrm{MHz}$ with different transmission power and protocol standards: ZigBee 2.4GHz $10 \mathrm{~mW}$ and XBee $868 \mathrm{MHz} 300 \mathrm{~mW}$

Four main zones of the Reserve were selected for the study: Ojillo (blue route in figure 1): is a forest zone, relatively plane; Santa Olalla (pink route in figure 1): lagoon zone very close to dunes with low density of vegetation; Humedales (red route in figure 1): completely open zone, more than $15 \mathrm{Km}$ of free obstacles floodplain, but completely dry area during our study; and Dunas (purple route in figure 1): this area is close to the sea. There are dunes that are shifting.

\section{RESULTS}

Figure 1 shows a google map of Doñana. The map shows several routes for the coverage study.

Two coverage tests have been performed for Texas ZigBee $2.4 \mathrm{GHz}$ and low power ZigBee transceivers. First one was done in a relatively open field (at Santa Olalla), and another on a tree zone (Ojillo). The maximum coverage range measured was around $250 \mathrm{~m}$ and $60 \mathrm{~m}$ respectively. This is due not only to the power of transmission but also to the absorption property of $2.4 \mathrm{GHz}$ signals versus $868 \mathrm{MHz}$ signals. $2.4 \mathrm{GHz}$ signal is more absorbed by vegetation than $868 \mathrm{MHz}$.

$868 \mathrm{MHz}$ XBee coverage study consisted in testing the efficiency of the link between two XBee transceivers at $868 \mathrm{MHz}$ and $300 \mathrm{~mW}$ transmission power. In the Palace of Doñana there is a 25 meters high tower. In this tower we set up the base-station for the measurements (bottom yellow point in figure 1). The base-station equipment was composed by one XBee mote connected to a laptop through USB and a software application written in $\mathrm{C \#}$ is used in order to manage information between motes. This software application contains functions, procedures and state machines to implement the communication through XBee. Once the software application is running, it sends a broadcast packet asking for mote discovery, and then it keeps waiting for answers. When at least one mote have been discovered, and using a software timer, the first mote will continuously be transmitting information using a point to point link to the just discovered mote, transmitting one packet per second. Furthermore, the XBee transceiver is able to make remote AT commands request, this feature is used by tower mote for requesting information about the power of last received radio packet for each timer overflow. Power radio reception measurement is returned to tower mote as a $\mathrm{dB}$ magnitude. Thank to the use of application software, we can characterize the quality and radio power of the transceiver links for the Doñana Biological Reserve scenario.

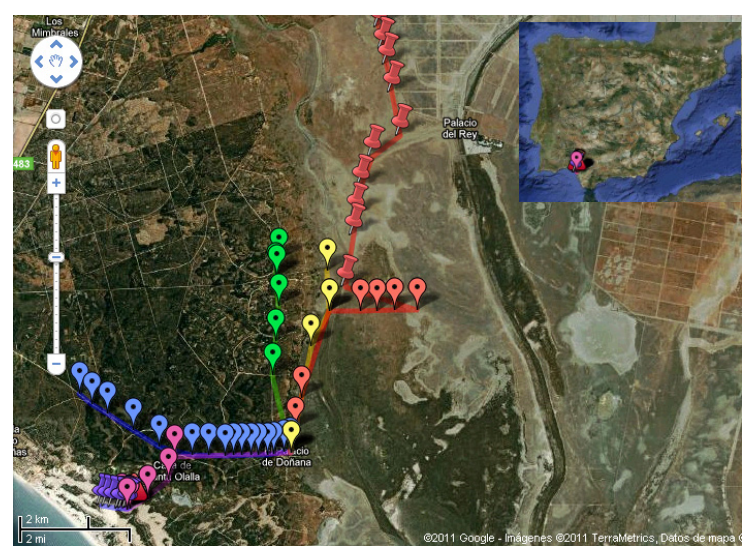

Figure 1: Google map of Biological Reserve of Doñana. Blue route from Palacio to Matalascañas route ended around Ojillo. Pink route from Palacio to Dunes. Purple route on Dunes. Green and red routes on Humedales.

The second mote consists on an XBee $868 \mathrm{MHz}$ transceiver at $300 \mathrm{~mW}$ connected to a microcontroller. The microcontroller is continuously retransmitting the same incoming packet. Therefore, the tower mote will receive the same transmitted packet with information about the signal intensity in $\mathrm{dB}$ thanks to the use of AT commands supported by XBee transceiver. During the different tested routes we have taken the GPS coordinates at each point of each route of figure 1. At these points we have measured the signal intensity in $\mathrm{dB}$ of the link on the tower side for two different position of the mote $(2 \mathrm{~m}$ high and $0.5 \mathrm{~m}$ high). Figure 2 shows a graph per each significant route. It can be seen that the signal is quite good for really open fields (Humedales), obtaining a coverage range of up to $13 \mathrm{Km}$, while for tree zones (Ojillo), the coverage rage decreases to $6 \mathrm{Km}$ and the signal intensity increases when measurements are made in a dense tree zone. At 
Dunas zone we realized that the signal is lost immediately when measuring from the tower mote (pink route), so we moved both XBee motes to Dunes zone and we obtained around $0.8 \mathrm{Km}$. Results of this zone are presented on figure 2 bottom.

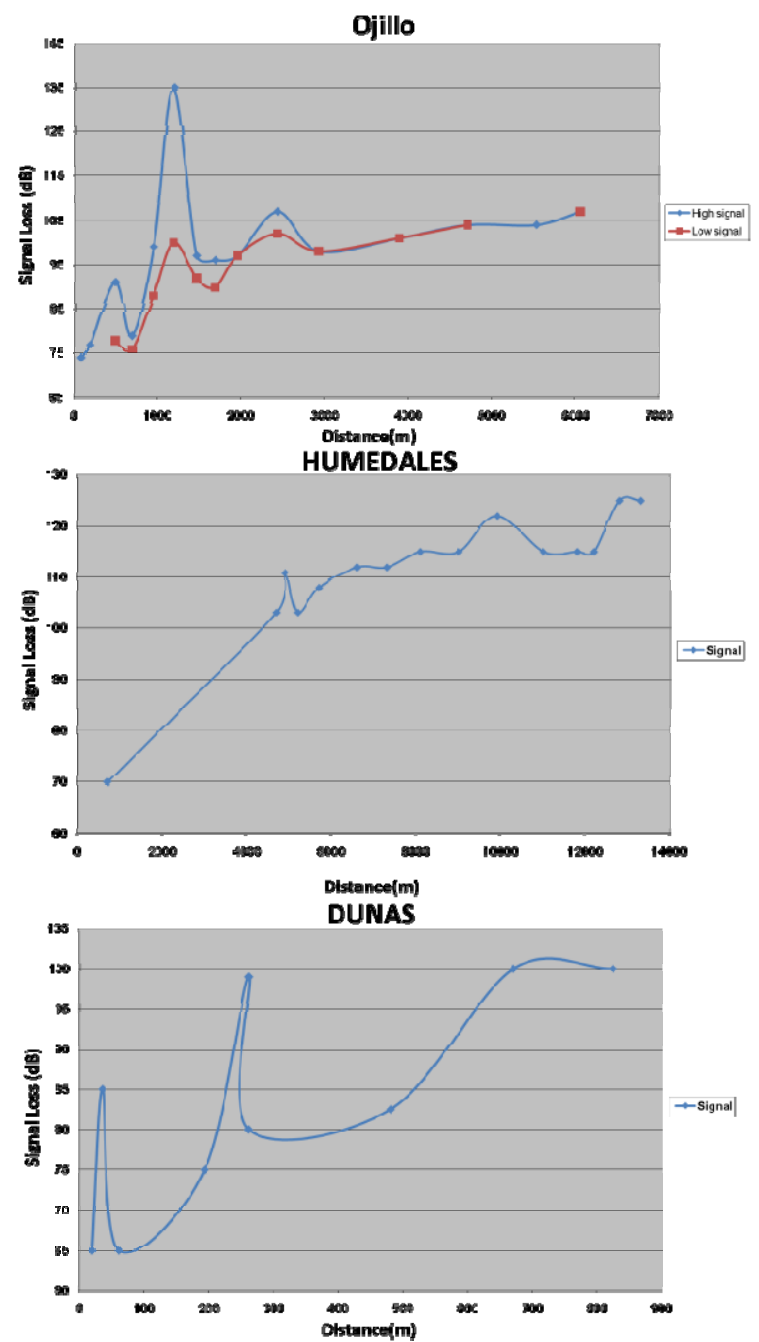

Figure 2: Signal reception in $\mathrm{dB}$ for Matalascañas route (blue on map), Humedales (wetlands) (red on map) and Dunes (purple on map).

\section{CONCLUSIONS}

This work presents a communications study in a free-building scenario (Doñana Biological Park). This study was carried out in various areas of the park (named above) to test how terrain and weather conditions changes affect the results. Due to the thick vegetation of the park, the signal emitted in 2.4 $\mathrm{GHz}$ was easily absorbed, reducing link distances and, therefore, significant results have been undergo using $868 \mathrm{Mhz}$ transmissions.
The equipment used, with $300 \mathrm{~mW}$ of power, reaches distances of several kilometres. In this way, natural habitat is not contaminated with lots of nodes through the park ( 2 or 3 points could cover DBR).

\section{ACKNOWLEDGEMENTS}

This work has been in part sustained by the RDBICTS 39/2010 Second Call Access 2009-2010 ICTSRBD (http://icts.ebd.csic.es/ListarConvocatoriasActi on.do)

\section{REFERENCES}

Ruedi Haller, Flurin Filli and Stephan Imfeld. 2001. Evaluation of GPS-technology for tracking mountain Ungulates: vhf-transmitters or GPS-collars? Tracking animals with GPS. Springer. ISBN: 0708406432

Robert S. Rempel, Arthur R. Rodgers and Kenneth F. Abraham. 1995. Performance of a GPS Animal Location System under Boreal Forest Canopy. Allen Press.

Philo Juang, Hidekazu Oki, et al. Energy-efficient computing for wildlife tracking: design tradeoffs and early experiences with ZebraNet. 2002. 10th international conference on Architectural support for programming languages and operating systems. $\mathrm{Pp}$ 96-107. ISBN:1-58113-574-2

Andrew C. Markham, Andrew J. Wilkinson. 2008. EcoLocate: A Heterogeneous Wireless Network Systemfor Wildlife Tracking. Novel Algorithms and Techniques in: Telecommunications, Automation and Industrial Electronics, Springer Science, Business Media B.V.

Scott L. Findholt, Bruce K. Johnson, Larry D. Bryant, JackW.Thomas, 1996. Corrections for Position Bias of a LORAN-C Radio-Telemetry SystemUsing DGPS. NoIhwest Science Vol. 70. No.3.

L. David Mech. 2002. A Critique of Wildlife RadioTracking and its Use in National Parks. A Report to the U.S. National Park Service. The Raptor Center, 1920 Fitch Ave., University of Minnesota, St. Paul, MN 55108.

Joseph Allen. 2009. Use of Coded Transmitter Schemes to Overcome Radio Frequency Spectrum Constraints in Terrestrial Wildlife Tracking. Report Advanced Telemetry Systems, Inc., Isanti, MN 55040

Vodafone Group Foundation. Introduction to Tracking Technology - GPS and GIS. Technologies for Conservation and Development, www.t4cd.org.

Doñana National Park. UNESCO. 1994. http://whc. unesco.org/en/list/685

Merrett, G. V., Weddell, A. S., Berti, L., Harris, N. R., White, N. M. and Al-Hashimi, B. M. 2008. A Wireless Sensor Network for Cleanroom Monitoring. In: Eurosensors, Dresden, Germany. 